\title{
A Study on Design of Health Device for U-Health System
}

\author{
Am-Suk Oh \\ Dept. of Media Engineering, Tongmyong University, Busan, Korea \\ asoh@tu.ac.kr
}

\begin{abstract}
U-health system supports health care services of prevention, diagnosis, medical treatment, post health management and currently various medical service systems such as remote diagnosis, emergency medical treatment, private living body monitoring are developed and serviced. There are network compatibility problems among the u-Health devices as their different protocols and software, which are manufactured depending on the characteristics of companies. The utilization of medical information thru hospitals and medical agencies is needed in this society. Thus, in this thesis, we study the design of health devices to analyze and adjust the U-health system.
\end{abstract}

Keywords: U-Health System, Health Device, Medical Information

\section{Introduction}

U-Healthcare technology can be used for diagnosis and medical treatment at any time, any place connected with networks for the aged and other patients. It is expected that the u-healthcare can solve the medical cost problem thru managing chronic diseases. Rapid development of information and communication technology also supports their health concern via remote diagnosis, emergency medical treatment and so on. Thus the communication system and interface system is considered an important element. Figure 1 shows general structure of private medical system, which is being used in U-Health.

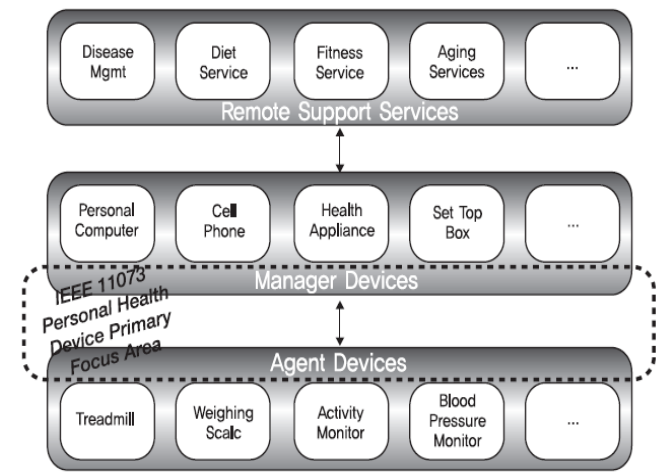

Figure 1. Architecture of Healthcare Devices

However, each individual healthcare device contains unique protocol and related software of each different company and those protocols are not opened to the public. Thus, there are a few data compatibility issues among such devices and the standardization to solve the problem becomes very important issue. Nevertheless, the standardization has hardly been done because of the increasing cost of the measuring- 
instruments and software. Furthermore, medical data of nonstandard format can't be interoperated or integrated into EHR without global standardization.

\section{ISO/IEEE 11073}

\subsection{IEEE 11073 PHD}

IEEE 11073 PHD is the standardization for exchanging information among private medical remote devices and managers. Those managers are mobile phones, computers, set top boxes, information collectors and so on.

Private healthcare devices can collect patient's information and transmits them to the manager such as a mobile phone, computer, or medical facilities. Managers can transmit the data to the remote supporting center for the detailed analysis. Normally, the communication channel among private healthcare devices and managers is carried out by point-to-point connection. Managers can communicate with multiple private devices using point-to-point protocol.

\subsection{PHD Standard Model}

11073 PHD standard models define protocol structure, private healthcare devices and $\mathrm{CE}$ and are constructed as 3 models that follow.

- Domain Information Model(DIM): It is Object-Oriented Model and defines object of private healthcare device. Each object has attributes of more than one and each attribute shows measurement data or function of device.

- Service Model: It defines access method between private healthcare devices and managers. Its access is defined in DIM whenever accessing the data. Service commands include GET, SET, ACTION, Event Report etc.

Communication Model: It defines network communication protocols by point-to-point connection. Generally, MDER (Medical Device Encoding Rule) is used as the coding of DIM in IEEE 11073 PHD.

\subsection{IEEE 11073 Protocol}

IEEE 11073 PHD includes protocol stack for the connection of private healthcare devices and managers. IEEE 11073 PHD protocol stack is structured and divided into 3 levels as follows.

- Transport Layer: IEEE 11073 PHD allows various transmission methods without detailed physical definition.

- Optimized Exchange Protocol: This level is the most important part in IEEE 11073 PHD. It provides supporting foundation of various private healthcare devices. Application service provides management of connection between private healthcare device and manager in addition to the reliable protocol. It also converts to Medical Device Encoding Rule(MDER), standard Binary ER(BER), Packet ER(PER) from ASN.

- Device Specialization: On the top of IEEE 11073 protocol stack, there is a device specialization layer which describes special detail items related to 8 private healthcare devices. This describes object and attributes of each private healthcare device.

\section{Design of Health Device}

The interface environment is characterized as Figure 2. It shows device interface between agents and managers, and it also shows the interface between manager and EDI. IEEE P11073-20601, which is composed of data exchange standard on application layer, 
defines logical session, data transmission, session disconnection on the view point of object.

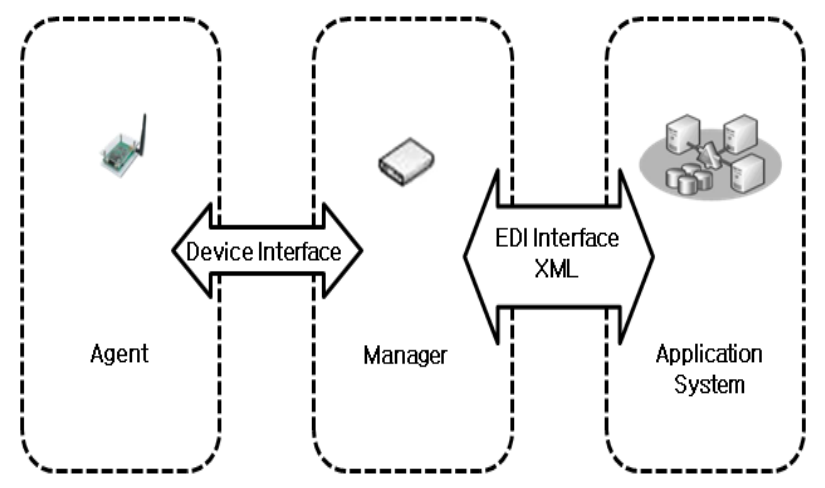

Figure 2. Interface Environment

\subsection{Device Specialization Standard}

IEEE 11073 PHD standard depending on Device specialization is as follows.

- 11073-10404: Device specialization-pulse oximeter

- 11073-10407: Device specialization-Blood Pressure

- 11073-10408: Device specialization-Thermometer

- 11073-10415: Device specialization-Weighing scale

- 11073-10417: Device specialization-glucose meter

- 11073-10441: Device specialization-Cardiovascular fitness and activity

- 11073-10442: Device specialization-Strength fitness equipment

- 11073-10471: Device specialization-Independent living activity hub

\subsection{PHD Standard Model}

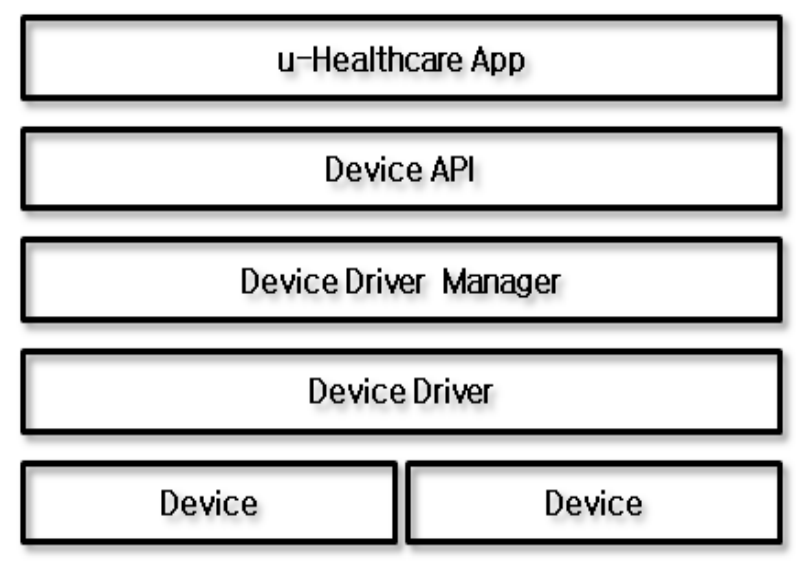

Figure 3. Device Interface Architecture

Figure 3 shows device interface architecture. Device interface can make connection applications and medical devices. It can also map applications and users in addition to the manipulation of device. Device API provides connection of application and Device Driver Manager. Device Driver supports the connection of Device Driver Manager and device. 


\subsection{Message Transmission Structure}

We designed event transmission structure and message based on HL7 for requesting and receiving medical information of hospitals, medical agencies. The designed structure is shown in figure 4.

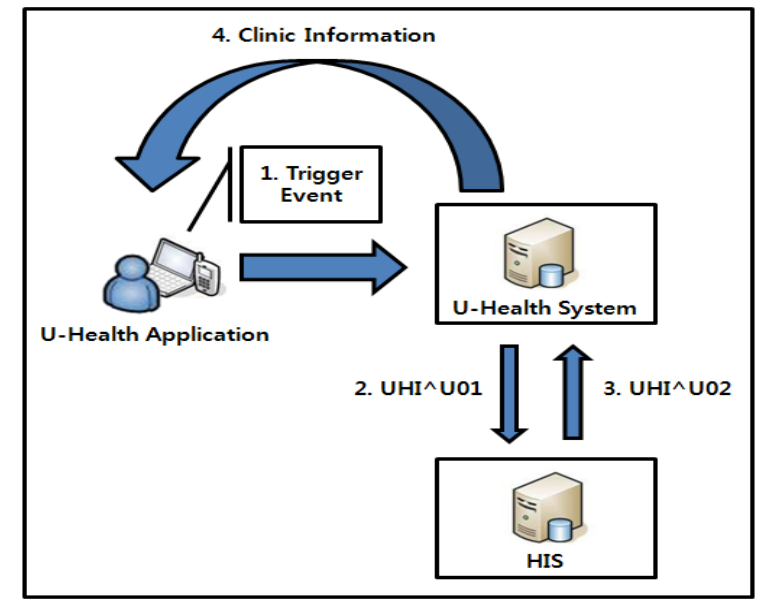

Figure 4. Message Transmission Structure

- Step 1: Making trigger event for the first information request thru health care user interface.

- Step 2: Request the patient's clinical information from U-Health system to HIS system.

- Step 3: Reply the patient's clinical data to U-Health system.

- Step 4: Share the prescribed patient's live body data with hospital.

\subsection{UHI Message Design}

We named medical information messages needed by system as UHI (Ubiquitous Healthcare Information). The requested message event from U-Health system to hospital HIS is defined as U01 and the reverse direction is defined as U02.

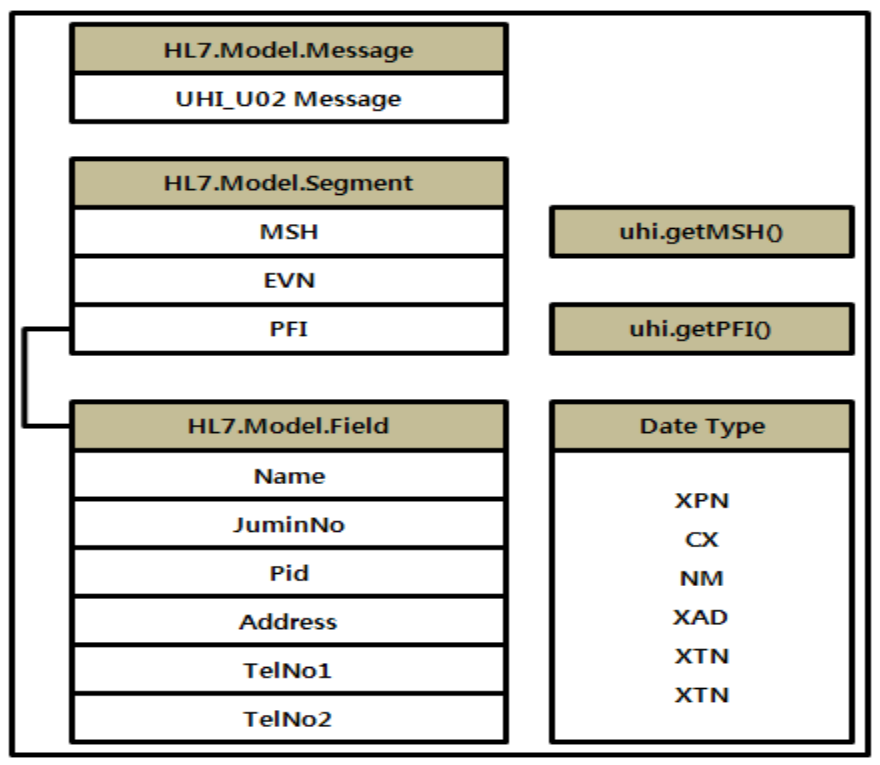

Figure 5. UHI^U01 Message Diagram 


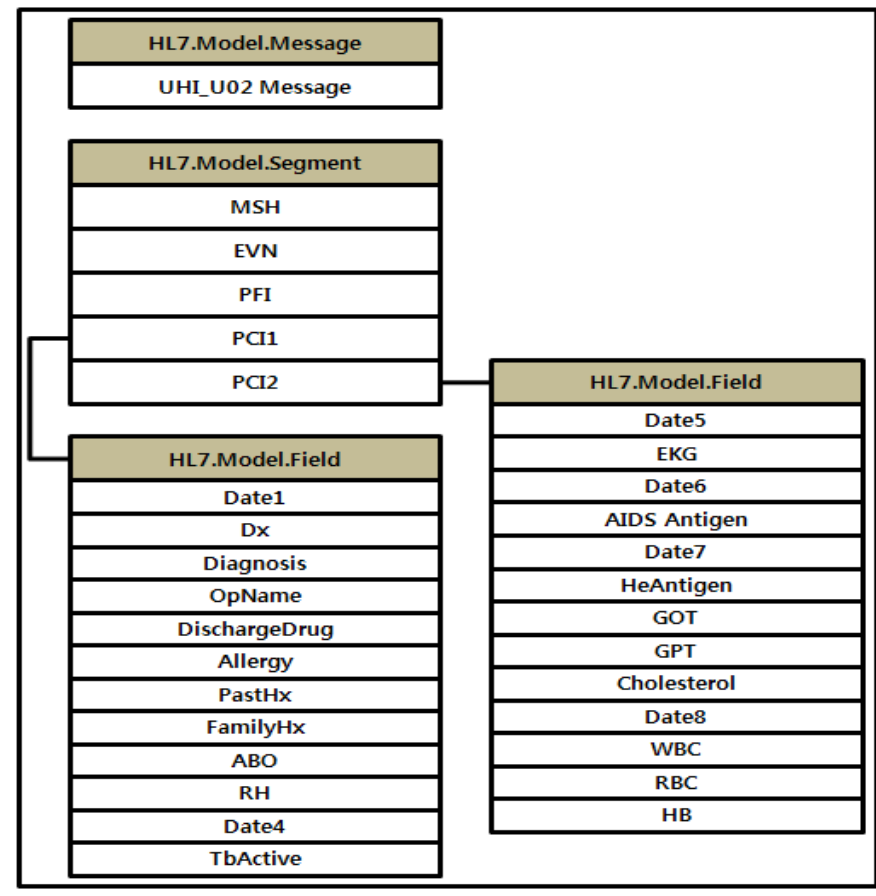

Figure 6. UH^^U01 Message Diagram

Figure 5 and 6 show the diagram of PCI (Patient Clinic Information) segments we designed and it can request or transmit medical information depending upon the field tag number. The contents of PFI and PCI segments which compose the UHI message and are considered as core part are as follows.

3.5. Design of PFI (Patient Foundational Information) segment: PFI segments designed for identification of patient who requested the information. Basically, it is designed to request patient's name, registered number, and additionally includes other information such as resident's ID, telephone number, address.

Table 1. PFI Segment Field

\begin{tabular}{||c|c|c||}
\hline \hline PFI & Field Name & Explain \\
\hline \multirow{4}{*}{$\begin{array}{c}\text { Foundation } \\
\text { Info. }\end{array}$} & Name & Patient Name \\
\cline { 2 - 3 } & JuminNo & Number \\
\cline { 2 - 3 } & Pid & Patient ID \\
\cline { 2 - 3 } & Address & Address \\
\cline { 2 - 3 } & TelNo1 & Telephone1 \\
\cline { 2 - 3 } & TelNo2 & Telephone2 \\
\hline
\end{tabular}

3.6. Design of PCI (Patient Clinic Information) segment: Medical information for U-Health system is basic patient's information defined as PID that is, standard HL7 segment and also special medical information which is used in main industry fields like remote diagnosis, realtime live body monitoring, emergency medical treatment is included. Special medical information setting on this design is transmitted to the field of PCI (Patient Clinic Information) segment[9]. 
Table 2. PCl1 Segment Field

\begin{tabular}{|c|c|c|}
\hline PCI1 & Field Name & Explain \\
\hline \multirow{15}{*}{$\begin{array}{l}\text { Clinic } \\
\text { Info. }\end{array}$} & Date1 & $\begin{array}{c}\text { Diagnosis \& } \\
\text { Prescription date }\end{array}$ \\
\hline & Dx & Diagnosis name \\
\hline & Diagnosis & Prescription name \\
\hline & Date2 & Date of surgery \\
\hline & OpName & operation name \\
\hline & Date3 & Discharge date \\
\hline & DischargeDrug & Discharge Drug \\
\hline & Allergy & Allergy \\
\hline & PastHx & Past medical history \\
\hline & DrugHx & Drug history \\
\hline & FamilyHx & $\begin{array}{l}\text { Family medical } \\
\text { history }\end{array}$ \\
\hline & $\mathrm{ABO}$ & ABO Blood \\
\hline & $\mathrm{RH}$ & RH Blood \\
\hline & Date4 & Date of TB test \\
\hline & TbActive & TB Active \\
\hline
\end{tabular}

\subsection{Design of Functional Module}

We designed an EDI Prototype that is contained in an EDI Converter and is in XML form for the efficient connection of various device and application system. This EDI Prototype can perform the function of data exchange among various devices and application systems depending upon each device type. Figure 7 shows EDI Converter Architecture.

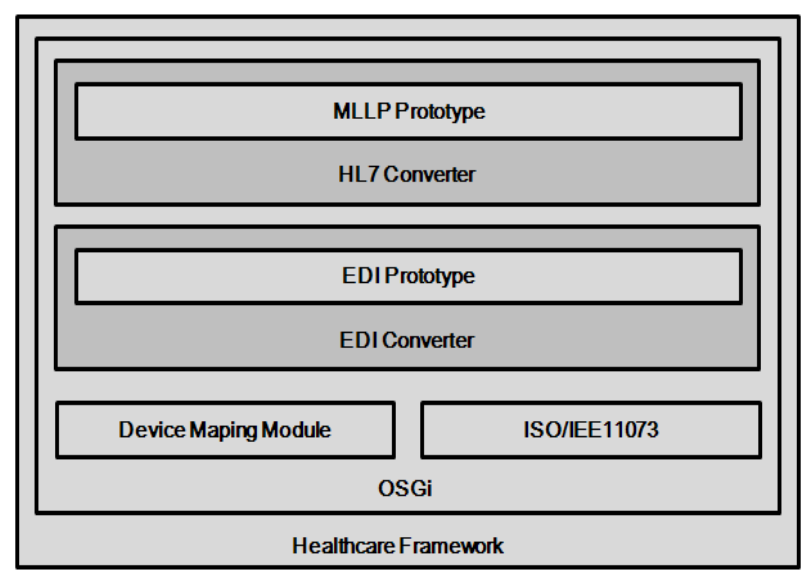

Figure 7. EDI Converter Architecture

We Service Model defines executive concept for the data exchange among agents and managers. For example, object access service of blood pressure gauge is used for accessing the object defined in domain information model of blood pressure gauge. It supports the following services. 
- GET service: It is used whenever managers get the object attributes of MDS(Medical Device System)

- SET service: It is used whenever managers set the object attributes of MDS.

- Event report service: It is used whenever an agent sends the measurement data and configuration report to the manager.

- Action service: It is used whenever managers- call the supporting action of agent.

\section{Conclusions}

Health devices do not support compatibility among other devices. Thus it disturbs UHealth system being activated. We studied health devices for U-Health system and designed it. It includes UHI message, functional module and EDI converter. Recently, health devices are operated with other medical systems such as diagnosis transmission system. The evolution of communication and ubiquitous technology especially make it faster. So, current legacy U-Health system is using health device and special medical information related to medical diagnosis, record of medical treatment. Thus, we designed health device for U-Health system under the U-Health service environment.

\section{References}

[1] Hapi HL7 SDK, (http://hl7api.sourceforge.net/).

[2] Unlocking the Power of Health Information, (http://www.hl7.org/).

[3] H. S. Oh, "u-Healthcare Technology and trand of Standard", IT Standard \& Test Journal, no. 112.

[4] Korea Food \& Drug Administration, "Standard of U-Health Medical Devices Guideline", (2007).

[5] C. S. Kim, "The Trends and Prospects of Health Information Standards: Standardization Analysis and Suggestions", Department of Radiological Science, College of Health Sciences, Catholic University of Pusan, (2008).

[6] B. Orguna and J. Vub, "HL7 ontology and mobile agents for interoperability in heterogeneous medical information systems", Computers in Biology and Medicine, vol. 36, (2006), pp. 817-836.

[7] T. S. Kim, “A Metadata System for HL7 aECG Document Management”, Department of Computer \& Information Engineering Graduate School, Chongju University, (2005).

[8] H. Park, "Implementation of Hospital Information System based on HL7 using RFID", Department of Electronics \& Communications Engineering Graduate School of Kwangwoon University, (2006).

[9] T. R. Lee, M. K. Choi and C. S. Yun, "Development and Implementation of System for Delivery of Emergency Patient's Basic Information between Related Hospitals", Journal of Health Science \& Medical Technology, vol. 29, no. 2, (2003), pp 67-80.

[10] K. H. Park and J. G. Pak, "Applying Remote Device Management Technologies to PHD-based uHealthcare Systems", Journal of Selected Areas in Communications, Unpublished observations, (2012).

[11] P. G. Pak and K. H. Park, "Efficient Message Encoding Method for Personal Health Device Monitoring System", Proc. of the 6th International Conference on Ubiquitous Information Management and Communication, (2012).

[12] W. W. Changa, T. J. Sungb, H. W. Huanga, W. C. Hsua, C. W. Kuoa and J. J. Changb, "A Smart Medication System using Wireless Sensor Network Technologies", Journal of Sensors and Actuators, vol. 172, no. 1, (2011), pp. 315-321.

[13] D. P. Bogia, "Supporting Personal Health Devices through Standardization and Collaboration", Proc. of the 13th IEEE International Conference on e-Health Networking Applications and Services, (2011).

[14] C. Y. Park, J. H. Lim and S. J. Park, "ISO/IEEE 11073 PHD Standardization of Legacy Healthcare Devices for Home Healthcare Services", Proc. of the IEEE International Conference on Consumer Electronics, (2011)

[15] M. Yuksel and A. Dogac, "Interoperability of Medical Device Information and the Clinical Applications: An HL7 RMIM based on the ISO/IEEE 11073 DIM", IEEE transactions on information technology in bio, vol. 15, no. 4, (2011).

[16] S. C. Nemesio, J. A. S. Cadenas and H. de Las, "OPENHEALTH - The Open Health FLOSS Implementation of the ISO/IEEE 11073-20601 Standard”, HEALTHINF - Proceedings, (2010). 


\section{Author}

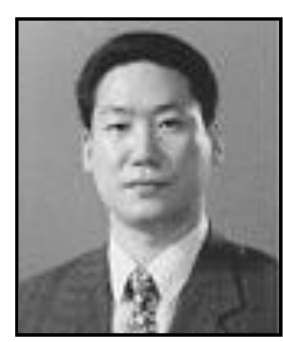

Am-Suk Oh, he received the B.S. and M.S. degrees in computer science from Busan National University and Chung-ang University, respectively. He received Ph.D degree at the computer engineering of Busan National University. He is currently with the Department of Media Engineering, Tongmyong University as Professor. His research interests are Database, Healthcare System, Medical Information System. 